\title{
Close-packed hemiellipsoid arrays: A photonic band gap structure patterned by nanosphere lithography
}

\author{
Wai Yuen Fu, Kenneth Kin-Yip Wong, and H. W. Choi ${ }^{\text {a) }}$ \\ Department of Electrical and Electronic Engineering, The University of Hong Kong, Hong Kong
}

(Received 23 April 2009; accepted 1 September 2009; published online 2 October 2009)

\begin{abstract}
A self-assembled hexagonal close-packed hemiellipsoidal photonic crystal structure was fabricated on GaN material. An ordered monolayer silica nanosphere coating served as a hard mask in an inductively coupled plasma etching process. The shape of the arrayed hemiellipsoids can be controlled by adjusting the etch selectivities and durations according to the fabrication model. The existence of a photonic band gap is established through planar transmissivity measurement whereby a transmission dip centered at $440 \mathrm{~nm}$ was identified. A threefold enhancement in light extraction was achieved, as determined from the measured angular photoluminescence emission pattern. (C) 2009 American Institute of Physics. [doi:10.1063/1.3238564]
\end{abstract}

In a typically configured light-emitting diode (LED) device structure grown on sapphire, as much as $80 \%$ of photons generated at the multiquantum wells (MQWs) are confined within the thick substrate and the GaN epilayer ${ }^{1}$ propagating as a guiding mode. Common techniques of boosting light extraction from a LED chip include surface texturing, ${ }^{2}$ integration of photonic crystal $(\mathrm{PhC}){ }^{3}$ and geometrical chip shaping. ${ }^{4}$ PhCs are particularly attractive, considering the multidimensional effects it is capable of offering. Most of the focus thus far has been devoted to $\mathrm{PhCs}$ patterned by electron beam lithography ${ }^{1}$ and nanoimprint lithography. ${ }^{5}$ Without a doubt, these lithographic techniques are capable of creating nanoscale patterns with high precision; their high fabrication cost renders them unfavorable for large-scale fabrication. In contrast, techniques involving self-assembly of colloidal nanoparticles, including nanosphere lithography adopted in this work, creates patterns with sufficiently good ordering at much lower costs and efficiency due to its parallel process nature. Reports on self-assembled PhC structures have mostly been experimental, ${ }^{6}$ and the mechanism of enhanced light extraction had been due to strengthening of coupling modes by the $\mathrm{PhC}$ 's diffractive behavior rather than through the existence of a photonic band gap (PBG). ${ }^{7}$

Indeed, self-assembled PhC structures do have their limitations. The major problem lies within the close-packed ordering of self-assembled patterns. For instance, the conventional nanopillar structure fabricated by direct transfer of the hexagonal closed-packed (hcp) nanosphere pattern onto optical materials possesses only a transverse magnetic (TM)mode PBG, which is not suitable for enhancing transverse electric (TE)-mode-dominated emission ${ }^{8}$ from LED structures. Therefore, the major objective is to find ways of introducing spacing of controllable dimensions between individual nanospheres, so as to relax the design constraints involving self-assembled PBG structures.

In this paper, we propose a self-assembled $\mathrm{PhC}$ structure based on a HCP hemiellipsoid (HE) array. The geometry of the HEs can be adjusted by controlling the process conditions (namely etch selectivity and duration), thereby tuning the PBG properties. The existence of a PBG and the feasi-

\footnotetext{
a) Author to whom correspondence should be addressed. Electronic mail: hwchoi@hku.hk. Tel.: (852) 2859 2693. FAX: (852) 25598738.
}

bility of our proposed structure in enhancing light extraction efficiency are demonstrated.

The proposed PBG structure was fabricated through a dual-step process, the process flow of which is illustrated in the schematic diagram of Fig. 1. A LED wafer, containing InGaN/GaN MQWs with emission centered at $440 \mathrm{~nm}$, was initially coated with plain 192-nm-diameter $\mathrm{SiO}_{2}$ nanospheres with coefficient of variation $(\mathrm{CV}) \sim 4 \%$ (acquired from Corpuscular Inc.) by vertical deposition method. The nanosphere solution, together with alcohol and sodium dodecyl sulfate, which accelerate the resulting solution's evaporation rate and reduce viscosity, respectively, were mixed in a vial. The wafer was immersed into the solution by laying it upon the sidewall of the vial, which was then placed into an oven maintained at a constant temperature of $40{ }^{\circ} \mathrm{C}$ with a stable supply of air stream for $3 \mathrm{hrs}$. The solution eventually evaporated, leaving the nanospheres self-assembled into a monolayer of HCP pattern on the surface of the wafer.

Subsequently, the coated sample was subjected to ICP dry etching for pattern transfer, during which the monolayer nanosphere coating served as a hard mask. A monolayer coating of nanosphere is thus essential for pattern transfer. In (a)
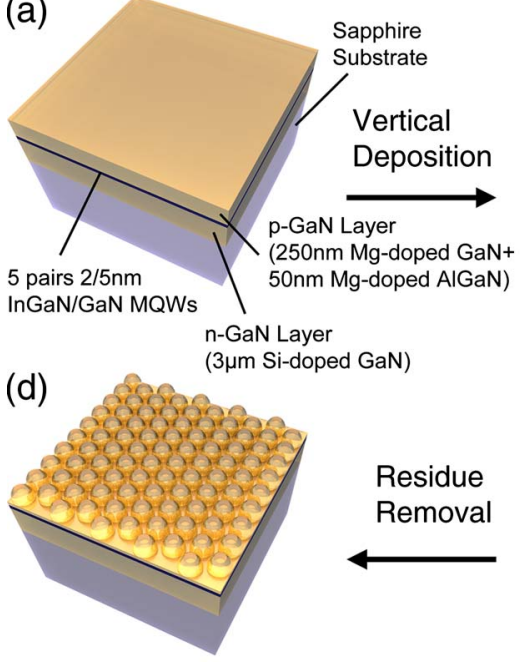

(b)

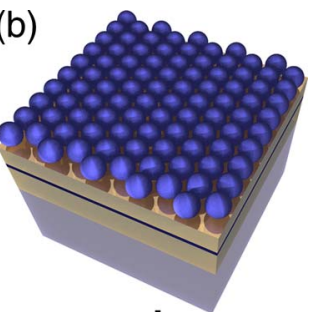
Dry Etch

(c)

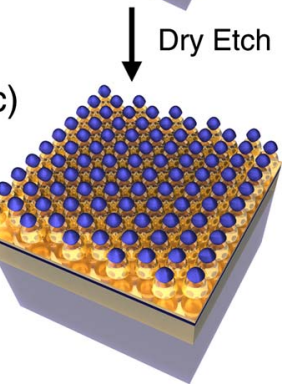

FIG. 1. (Color online) Schematic diagram detailing the fabrication process (a) LED wafer, (b) the wafer coated with a monolayer of plain silica nanosphere, (c) residue of nanosphere with etched pattern beneath during etching, and (d) the PBG structure formed after removing the residue. 


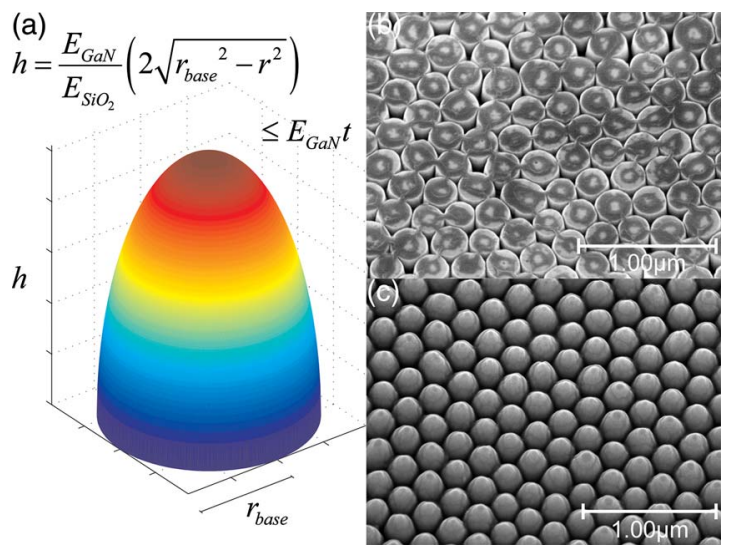

FIG. 2. (Color online) (a) HE fabrication model. (b) shows a nanopillar array with slightly inclined sidewall, after etching for $120 \mathrm{~s}$. (c) The final arrayed HE PBG structure formed when etching time increased to $290 \mathrm{~s}$.

our previous works, the coated samples were etched using a highly selective recipe comprising $\mathrm{Cl}_{2}$ and Ar-producing HCP nanopillars with near-vertical sidewalls. ${ }^{3}$ In this work $\mathrm{CHF}_{3}$ was added to the gas chemistry, offering appreciable etch rates to $\mathrm{SiO}_{2}$. Due to the isotropic nature of the etch recipe, together with the introduction of $\mathrm{CHF}_{3}$ for etching $\mathrm{SiO}_{2}$, the lateral dimensions of the nanospheres shrink as etching progresses. In fact, the shape of the resultant nanostructure etched for a duration of $t$ can be deduced according to the equations and diagram in Fig. 2(a), with $E_{\mathrm{GaN}}$ and $E_{\mathrm{SiO}_{2}}$ being the etch rates of $\mathrm{GaN}$ and $\mathrm{SiO}_{2}$, respectively.

The HE structure was progressively shaped during the dry etch process. The field-emission scanning electron microscope image in Fig. 2(b) illustrates nanopillars with slightly inclined sidewalls formed after etching for $120 \mathrm{~s}$, at gas flow rates of 12 SCCM (SCCM denotes cubic centimeter per minute at STP) for $\mathrm{Cl}_{2}$ and $9 \mathrm{SCCM}$ for $\mathrm{CHF}_{3}$, respectively. Based in this recipe, the etch selectivity between $\mathrm{GaN}$ and $\mathrm{SiO}_{2}$ was approximately 4:3. The final required structure was obtained after a total etch duration of $290 \mathrm{~s}$, as shown in Fig. 2(c). The nanosphere residue was removed by $10 \mathrm{~min}$ of wet etch in $10 \%$ hydrofluoric acid followed by $10 \mathrm{~min}$ of sonication in acetone. The height of the resulting structure is roughly $250 \mathrm{~nm}$, leaving a gap of about $50 \mathrm{~nm}$ between the $\mathrm{PhC}$ and the MQWs. The parameters were chosen such that the depth of HEs would not penetrate the MQWs, and the PBG position should coincide with the emission wavelength of the LED structure, i.e., $440 \mathrm{~nm}$.

The PBG structure was designed using the software RSoft BANDSOLVE, which adopts the plane wave expansion approach. The vector Helmholtz equation was solved with the periodic PBG structure being modeled by Bloch's theorem. ${ }^{9}$ The supercell technique was employed to account for the varying dimension along the $h$ axis. The simulated TE and TM band structures are plotted in Figs. 3(a) and 3(b), respectively. As observed from Fig. 3(a), there exists a PBG between $\sim 0.400$ and 0.450 within the light line of air, corresponding to wavelengths of about 420 to $480 \mathrm{~nm}$, considering the periodicity of the structure to be the diameter of a nanosphere, i.e., $192 \mathrm{~nm}$. Based on the same parameter, the TM PBG is predicted to be within the near-ultraviolet (UV) region, and can thus be neglected due to the inherent nature of InGaN MQW devices whereby TE modes dominate more than $80 \%$ of its overall emission. ${ }^{8}$ The region outside the light line can also be ignored as it represents the portion of
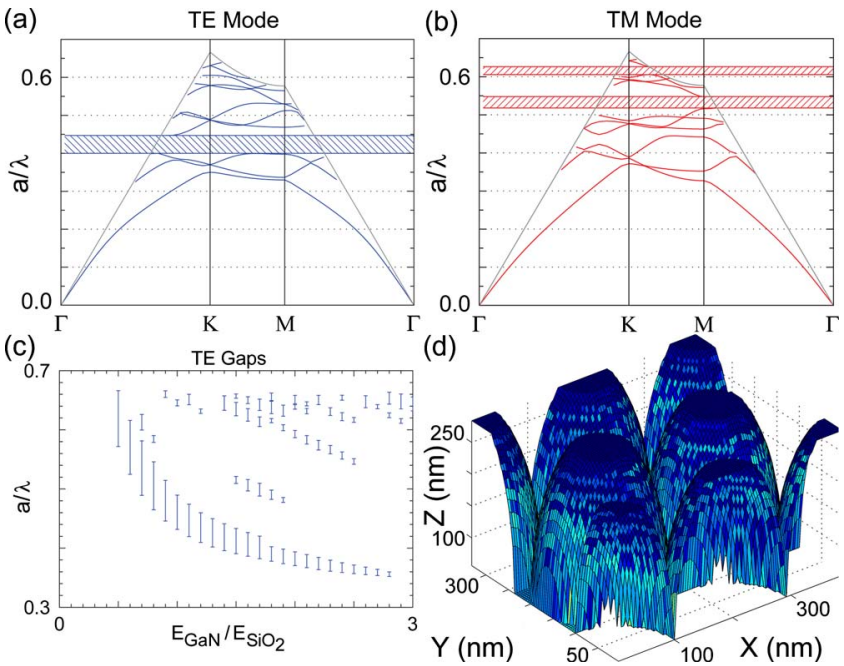

FIG. 3. (Color online) Simulated (a) TE and (b) TM band structure. (c) Plot of simulated TE PBG vs etch selectivity. (d) Results of FDTD simulation showing energy flux distribution across the HE array structure.

light directly extracted from $\mathrm{GaN}$ to air. Moreover, based on the model described in Fig. 2(a), the geometry of the PBG structure will vary according to the etch selectivity and duration. The position of the PBG will also shift accordingly. A plot of the simulated PBG location as a function of etch selectivity $\left(E_{\mathrm{GaN}} / E_{\mathrm{SiO}_{2}}\right)$ (for a constant feature height of 250 $\mathrm{nm}$ ) is presented in Fig. 3(c). As the selectivity increases, the fundamental TE PBG varies between 0.64 and 0.37 . With the 192-nm-diameter nanospheres used in this study, the tuning range of PBG position can potentially be up to $200 \mathrm{~nm}$.

A finite-difference time-domain (FDTD) simulation was also carried out to predict the energy flux distribution across the PBG structure. The simulation, coded in MATLAB, was set to have a dipole excitation source emitting at $440 \mathrm{~nm}$, with symmetric boundary in all directions except in the upward direction, where the perfectly matched layer was implemented. Figure 3(d) is the accumulated energy flux distribution sampled in the air medium just above the GaN structure, which represents the near field extracted light energy flux. The result shows that most of the energy is extracted at the bottom of the HE structure, and also from the triangular area among the HE. Such periodic spatial emission pattern suggests the diffractive nature of light emission from the PhC structure. Also, the results show $88.6 \%$ of guided modes in the $p$-GaN layer are coupled out.

To verify the existence of a PBG, a planar transmission measurement was conducted. Light emitted from a broadband tungsten-halogen lamp was collected by a fiber. After being collimated by two plano-convex lenses, the beam was focused by a $20 \times$ magnification UV objective. With slight downward inclination, the beam was directed onto the sample exciting guiding modes within the wafer, resulting in three output beam spots, corresponding to the three guiding modes along the $p$-GaN, $n-\mathrm{GaN}$, and sapphire layers. The output light beam corresponding to the guiding mode between the $p-\mathrm{GaN}$ layer and $\mathrm{MQW}$, where the PBG structure is situated, was then collected and coupled by another optical fiber to a spectrometer. Figure 4(a) shows the measured transmission spectrum. A pronounced transmission dip centered around $440 \mathrm{~nm}$ with a full width at half maximum of $55 \mathrm{~nm}$ was observed, correlating well with the simulated results, testifying to the existence of a PBG. The slight de- 

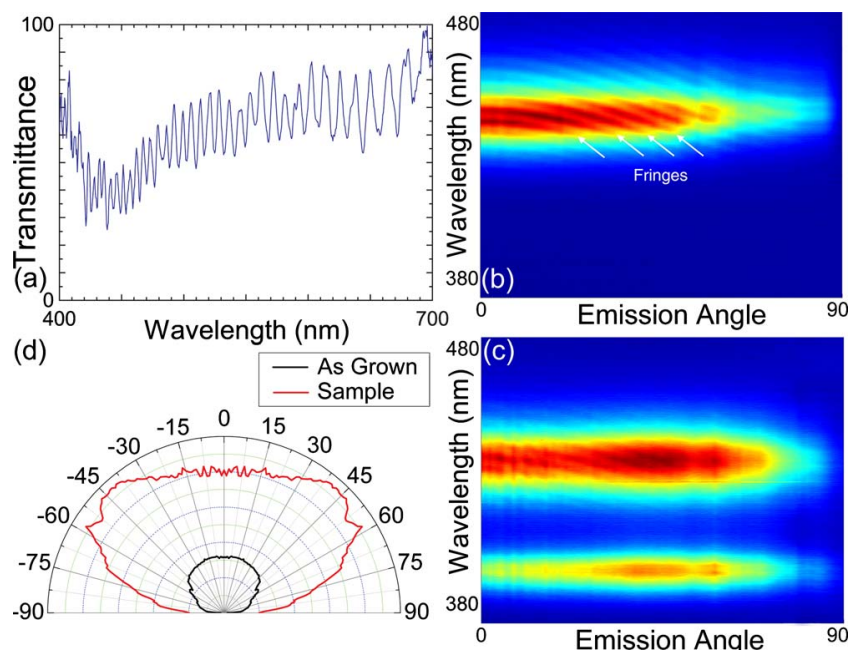

FIG. 4. (Color online) (a) Measured transmission spectrum, with reference to a GaN LED as-grown sample, measured and normalized angle-resolved PL spectrum for (b) as-grown sample and (c) PBG structure, and (d) measured PL angular emission pattern from the PBG structure.

viation may be attributed to defects, which include line defects, point defects, and shape irregularities that exist within the self-assembled $\mathrm{PhC}$, and possibly due to size deviation of nanospheres from the expected value, although these defects may actually contribute to better coupling efficiency due to the introduction of defect modes. Besides, fringes in the spectrum are attributed to the Fabry-Perot (FP) modes within the GaN layer in the reference spectrum taken from an as-grown sample. The maximum transmission was below $100 \%$, probably due to light scattering caused by the $\mathrm{PhC}$ structure.

A PBG structure integrated into a LED promotes light extraction efficiency in two major ways. The presence of a PBG in the direction of the plane suppresses laterally propagating guiding modes, channeling them into the vertical direction across the $\mathrm{GaN}$-air interface. It also gives rise to a surface texturing effect. Angle-resolved photoluminescence (PL) measurements were carried out to evaluate the possible effects of a PBG structure on the emission pattern. The beam from a third harmonic neodymium: yttrium lithium fluoride (Nd:YLF) diode-pumped solid state laser from SpectraPhysics at wavelength of $349 \mathrm{~nm}$ was focused onto the sample at an oblique angle to excite the MQWs beneath. The high absorption UV photons in GaN prohibits light guiding, restricting the excitation to a tiny spot. The emitted light was then collected by a fiber at a constant separation of $20 \mathrm{~mm}$ directly on top of the excited spot, at angles varying from $0^{\circ}$ to $90^{\circ}$ at a $1^{\circ}$ interval. The measured angular PL emission pattern and angle-resolved PL spectrum are presented in Fig. 4.

According to Fig. 4(d), a significant overall increase in light extraction efficiency was observed, with an enhancement factor of more than 3 , computed by comparing the integrated PL intensities of the emission patterns of the patterned and as-grown samples. The light intensity for the asgrown sample starts to drop as it approaches the critical angle of $\mathrm{GaN}$ and air (of $23^{\circ}$ ), while the sample with the PBG structure maintains considerable light intensity up to $60^{\circ}$. This is attributed to surface texturing effect brought about by the PhC geometry, since the array of HEs acts as an effective medium for light to escape, producing a batwing emission pattern. This is consistent with the FDTD simula- tion of Fig. 3(d) that light emission will be highly diffracted. Also observed in Fig. 4(b), the angle-resolved PL spectrum of the as-grown sample contains fringes in the diagonal direction. These fringes correspond to shifts in the FP modes due to optical oscillations in the GaN layer. Similar fringes in Fig. 4(c) are significantly suppressed as the FP modes are annihilated due to the surface texturing effect introduced by the PBG structure. On top of this, light extraction is further enhanced via the PBG. Vertical fringes observed between $0^{\circ}$ and $20^{\circ}$ in Fig. 4(c) can be attributed to the diffraction due to PhC. No emission lines are exhibited in Fig. 4(c), since the PBG covers a wavelength range of 420 to $480 \mathrm{~nm}$, as demonstrated in Figs. 3(a) and 4(a).

Secondary effects observed from the PL spectrum are also associated with the surface nanostructuring. First, the primary emission peak exhibits a spectral blueshift of $10 \mathrm{~nm}$, attributed to higher effective optical pumping density caused by the enhanced light coupling efficiency after implementing the PBG structure, despite the optical pumping densities at the source being unchanged for both measurements. Additionally, an extra emission peak is observed at about $395 \mathrm{~nm}$, due to exposure of the $\mathrm{Mg}$-doped AlGaN current blocking layer embedded between the MQW and $p$-GaN layer after etching.

Even with a threefold enhancement in light extraction achieved, there is further room for improvement by optimizing light coupling between the MQW and the PBG structure, which has not been addressed in this paper. The etch depth was intentionally chosen to minimize damage to MQWs by from plasma exposure but a deeper penetration extending beyond MQW region may form a nanoresonant cavity which can improve the Purcell factor as a result of a greatly reduced effective volume, and a high cavity quality factor due to PBG. The HE geometry presented in this work distinguishes from other common flat-topped $\mathrm{PhC}$ structures in terms of light extraction, bearing resemblance to the highly effective hemispherical encapsulation albeit on a nanoscale.

In summary, enhanced light extraction with a selfassembled arrayed HEs PBG structure has been demonstrated. The shape of the self-assembled array can readily be controlled through adjusting the etching parameters, and thus resulting in tunability of the PBG position. Through optical transmission and angle-resolved PL experiments, the predicted TE-mode PBG is verified, which was proven to play a vital role in promoting light extraction.

\footnotetext{
${ }^{1}$ A. David, T. Fujii, R. Sharma, K. McGroddy, S. Nakamura, S. P. DenBaars, E. L. Hu, C. Weisbuch, and H. Benisty, Appl. Phys. Lett. 88, 061124 (2006)

${ }^{2}$ D.-S. Han, J.-Y. Kim, S.-I. Na, S.-H. Kim, K.-D. Lee, B. Kim, and S.-J. Park, IEEE Photonics Technol. Lett. 18, 1406 (2006).

${ }^{3}$ W. N. Ng, C. H. Leung, P. T. Lai, and H. W. Choi, Nanotechnology 19 , 255302 (2008).

${ }^{4}$ W. Y. Fu, K. N. Hui, X. H. Wang, K. K. Y. Wong, P. T. Lai, and H. W. Choi, IEEE Photonics Technol. Lett. 21, 1078 (2009).

${ }^{5}$ K. J. Byeon, S. Y. Hwang, and H. Lee, Appl. Phys. Lett. 91, 091106 (2007).

${ }^{6}$ A. J. Danner, B. Z. Wang, S. J. Chua, and J. K. Hwang, IEEE Photonics Technol. Lett. 20, 48 (2008).

${ }^{7}$ S. H. Fan, P. R. Villeneuve, J. D. Joannopoulos, and E. F. Schubert, Phys. Rev. Lett. 78, 3294 (1997).

${ }^{8}$ P. G. Eliseev, G. A. Smolyakov, and M. Osinski, IEEE J. Sel. Top. Quantum Electron. 5, 771 (1999).

${ }^{9}$ J. D. Joannopoulos, S. G. Johnson, J. N. Winn, and R. D. Meade, Photonic Crystals: Molding the Flow of Light, 2nd ed. (Princeton University Press, Princeton, 2008).
} 